\title{
Study of memory effect in an economic order quantity model with quadratic type demand rate
}

\author{
R. Pakhira ${ }^{1}$, U. Ghosh ${ }^{2}$, S. Sarkar ${ }^{3}$ \\ University of Calcutta \\ Department of Applied Mathematics \\ Kolkata, 700009 \\ West Bengal, India \\ ${ }^{1}$ E-mail: rituparna.pakhira@gmail.com \\ ${ }^{2}$ E-mail: uttam_math@yahoo.co.in \\ ${ }^{3}$ E-mail: susmita62@yahoo.co.in
}

Received: 10 February 2019; revised: 07 June 2019; accepted: 10 June 2019; published online: 24 June 2019

\begin{abstract}
The study of memory effect in an economic order quantity model has a great impact on the inventory system. Although business policy almost depends on the past experiences of the system, usually the classical inventory model does not include the past experience or memory effect, i.e. one important part of the system is ignored. Our purpose is to include memory or past experience in the inventory model. The purpose of this paper is to incorporate the existence of dynamic memory in an inventory model with shortage via fractional calculus. To derive the memory dependent inventory model associated with inventory holding cost, shortage cost has been developed. Analytical solution of the proposed inventory model has been solved via primal geometric programming method. Numerically long memory effect or short memory effect of the inventory system has been established. In this paper, an effort has also been made to compare the memory effect on the minimized total average cost and the optimal ordering interval using different numerical examples.

Key words: fractional Laplace transforms method, differential equation with memory kernel, classical inventory model, memory dependent Inventory model
\end{abstract}

\section{INTRODUCTION}

The origin of the fractional calculus goes back to L'Hospital and Leibnitz in the seventeenth century. The modern work devoted exclusively to the subject of fractional calculus has been published [1-8]. Since that moment many monographs dedicated to this subject have been published [1-8]. Until the present arms fractional calculus have been discussed as a comparatively esoteric mathematical theory without applications but in the recent few years fractional calculus has appeared with its application backgrounds in different mathematical problems [1, 5, 6, 22-24], Physics [8], Economics [2-5], etc. Many well-known scientists like Leibniz, Euler, Lagrange, Fourier, Abel, Liouville,
Riemann, Grunwald, Holmgren, Peacock, Tarday, Cauchy, Hadamard, Hardy, Riesz, Weyl, etc. [9] worked on the fractional calculus to develop fractional derivative and integration. Fractional derivative and integration are generalized as non-integer order. Riemann-Liouville (R-L) fractional integration, Caputo fractional derivative $[9,10]$, Jumarie fractional derivative $[9,10]$ play a crucial role in developing any real life problems. It is worth noting that the standard classical mathematical models of the integer order derivatives do not work as a generalized version, but as a particular case of generalized version and are also not able to take into account memory of the system.

On the other hand, the first order ordinary derivative is equivalent to the physical meaning of speed (the physical 
meaning of speed is path length traveler per unit of time). But the physical interpretation of the fractional order derivative and integration is an index of memory [1]. In the present paper, the fractional calculus has been applied to illustrate the memory effect of the inventory system. The authors believe that the research in the area of the fractional calculus will constitute an important tool in the scientific progress of mankind.

It is known that having the ability to measure inventory in a time with accurate policy is critical without arranging the inventory model from a company's balance sheet chart. Owing to the above problem, inventory literature was born by a cited article of Harris-Willsons [11, 12] square root formulae. Most of these inventory models are proposed on the economic order (EOQ) model by many researchers [13-16]. In this paper, the classical inventory model has been developed on the basis of the fixed shortage time. The objective of this paper is to generalize the classical model using fractional derivatives and fractional integration as well as taking into account memory effect of the inventory model. Authors expect that memory dependent inventory model has more importance compared to the memory less inventory model or classical model.

In a real market or real inventory system the demand of the product changes with respect to time as well as space or situation. It is known that when an object gets popularity in the market then its demand will increase or if it makes bad impression then its demand will decrease. In some sense demand of any object is not the same in all shops: increase or decrease of the selling depends dealings of the shopkeeper, i.e. the selling of any product depends on the quality as well as the shopkeeper's attitude. Thus, this system depends on previous history, not only on the present state of the process, so it is legal to include the memory effect in the inventory system. We think that the memory effect should be incorporated in different human related physical problems and observe the different situation for low or strong memory effect or long memory effect, short memory effect.

Here, we have suggested fractional order derivative to take into account the memory effect. But why?

It is known that the time rate of change of integer orders is determined by the property of differentiable functions of time only in infinitely small neighborhood of the considered point of time. Hence, an instant change of the marginal output is assumed, when the input level changes. Therefore, the dynamic memory effect is not present in classical calculus and it is not able to discuss all state of the system i.e. (the present system depends on the past) [1-7]. But in fractional derivative the rate of change is affected by all points of the considered interval, so it is able to incorporate memory of the system and fractional order is physically treated as an index of memory. So, it can remove amnesia from the system.

Our analysis has established the existence of the memory effect in the EOQ model. It is observed from the numerical example that in the long memory effect the minimized to- tal average cost is very low compared to the memory less or short memory effect. In the long memory effect, the optimal ordering interval is much higher compared to the short memory effect. Hence, in the long memory effect business takes much more time to reach the minimum value of the total average cost compared to the short memory effect. When the demand rate is proportional to square of the time, the minimized total average cost with the memory effect is low compared to the quadratic type demand rate, constant demand rate, and linear type demand rate. Hence, when the system is strong with its past experience, profit is maximum compared to short gained past experience.

The rest part of the paper has been furnished as follows, In Sec. II., the review of fractional calculus has been discussed, the classical inventory model has been given in Sec. III., the memory dependent inventory model with analytic calculation has been proposed in Sec. IV., numerical examples and sensitivity analysis have been conducted in Sec. V., graphical presentation has been arranged in Sec. VI., and finally some conclusions are given to show the effect of the work in Sec. VII.

\section{REVIEW OF FRACTIONAL CALCULUS}

There are several definitions of the fractional derivative, the mostly used to definitions are given below.

\section{1. Riemann Liouville fractional derivative}

Left Riemann-Liouville (R-L) definition of fractional derivative of $\alpha^{\text {th }}$ order (where $m \leq \alpha<m+1$ ) for any integrable function $f(x)$ on $[a, b]$ is denoted ${ }_{a}^{\mathrm{RL}} D_{x}{ }^{\alpha}(f(x))$ and defined as

$$
\begin{aligned}
& { }_{a}^{\mathrm{RL}} D_{x}{ }^{\alpha}(f(x))= \\
& =\frac{1}{\Gamma(m+1-\alpha)}\left(\frac{d}{d x}\right)^{m+1} \int_{a}^{x}(x-\xi)^{(m-\alpha)} f(\xi) d \xi .
\end{aligned}
$$

The above results create a difference between ordinary derivative and fractional derivative. To eradicate this difficulty, M. Caputo $[9,10]$ proposed a new definition of fractional order derivative for any differentiable functions.

\section{2. Caputo fractional order derivative}

For any differentiable function $f(x)$ on $[a, b]$ Caputo fractional order derivative [15] of $\alpha^{\text {th }}$ order is denoted ${ }_{a}^{\mathrm{C}} D_{x}^{\alpha}(f(x))$ and defined as

${ }_{a}^{\mathrm{C}} D_{x}^{\alpha}(f(x))=\frac{1}{\Gamma(m-\alpha)} \int_{a}^{x}(x-\xi)^{(m-1-\alpha)} f^{(m)}(\xi) d \xi$,

where: $m-1 \leq \alpha<m$.

The Caputo definition contains both merit and demerit. Demerit of this definition is that the function should be $(m)$ times differentiable; otherwise this definition will not be valid. On the other hand, merit of this definition is, Caputo 
fractional order derivative of any constant function is zero as in ordinary derivative. In the main work of the paper, the Caputo fractional derivative operator has been symbolized as ${ }_{0}^{\mathrm{C}} D_{t}^{\alpha}$.

\section{3. Fractional Laplace Transform Method}

Laplace transformation plays an important role in integer and fractional order differential equations. The Laplace transform of the function $[9,10] f(t)$ is denoted by $F(s)$ and defined as

$$
F(s)=L(f(t))=\int_{0}^{\infty} e^{-s t} f(t) d t
$$

where $\mathrm{s}>0$ and $s$ is called the transform parameter. The Laplace transformation of integer order derivative is defined as

$$
L\left(f^{m}(t)\right)=s^{m} F(s)-\sum_{k=0}^{m-1} s^{m-k-1} f^{k}(0),
$$

where $f^{m}(t)$ denotes $m^{\text {th }}$ order ordinary derivative of $f$ with respect to $t$. For non-integer order $\alpha$, it is defined in generalized form as

$$
L\left(f^{\alpha}(t)\right)=s^{\alpha} F(s)-\sum_{k=0}^{m-1} s^{k} f^{\alpha-k-1}(0),
$$

where $(m-1)<\alpha \leq m$.

\section{4. Differential equation with memory kernel}

If we consider any first order differential equation in the form as

$$
\frac{d\left(f_{1}(x)\right)}{d x}=-f_{2}(x)
$$

then in terms of the memory kernel it can be written as follows [1]

$$
\frac{d\left(f_{1}(x)\right)}{d x}=-\int_{a}^{x} K(x-\xi) f_{2}(\xi) d \xi .
$$

To derive the concept of memory effect via fractional calculus, we consider $K(x-\xi)=\frac{(x-\xi)^{(\alpha-2)}}{\Gamma(1-\alpha)}$ and substitute it in (7) and we get

$$
\begin{aligned}
& \frac{d\left(f_{1}(x)\right)}{d x}=-\int_{a}^{x} \frac{(x-\xi)^{(\alpha-2)}}{\Gamma(1-\alpha)} f_{2}(\xi) d \xi= \\
& =-{ }_{a} D_{x}^{-(\alpha-1)}\left(f_{2}(x)\right) .
\end{aligned}
$$

Now, taking both sides $(\alpha-1)$ derivative and we get the required fractional order memory dependent differential equation

$$
{ }_{a} D_{x}^{\alpha}\left(f_{1}(x)\right)=-f_{2}(x)
$$

Here, the rate of memory kernel decays depending on the fractional parameter $\alpha$. The strength of the memory is controlled by the fractional order $\alpha$. When $\alpha \rightarrow 1$, the system becomes week in the sense of memory and when $\alpha=1$, the system becomes memory less.

Long Memory: The strength of memory is controlled by the order of fractional derivative or fractional integration. If the order of fractional derivative or fractional integration lies in the range $(0,0.5)$, then the system may be called to have long memory effect.

Short Memory: If the order of fractional derivative or fractional integration is in the range $[0.5,1)$, then the system is affected by short memory.

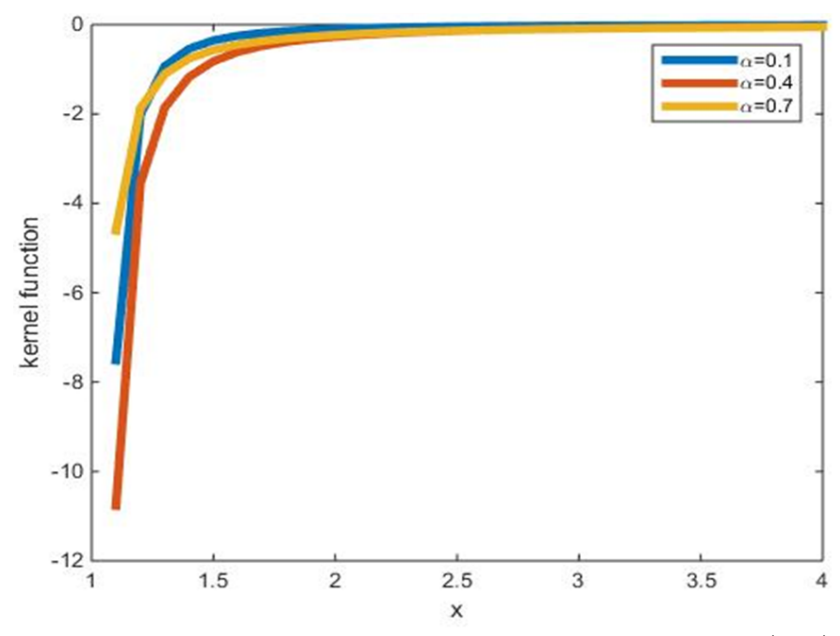

Fig. 1. Plot of memory kernel function $\left(k(x-\xi)=\frac{(x-\xi)^{(\alpha-2)}}{\Gamma(1-\alpha)}\right.$, for $\xi=3$ ) for different value of $\alpha$

It is clear from Fig. 1 that the pick of the curve gradually decreases depending on $\alpha$. Hence, memory strongly depends on the fractional order $\alpha$.

\section{CLASSICAL MODEL}

The classical or memory less inventory model has been developed [13] on the basis of the assumptions below. First, we have formulated the classical inventory model.

\section{1. Assumptions}

Here, the following assumptions are made in developing the inventory model.

- The inventory consists of only one type of items.

- The lead time is zero or negligible.

- Demand rate is $\left\{\begin{array}{l}\left(a+b t+c t^{2}\right) \text { for } 0 \leq t \leq t_{1} \\ D_{0} \text { for } t_{1} \leq t \leq T\end{array}\right.$

- The planning horizon is infinite.

- Shortage occurs during $t_{1} \leq t \leq T$.

- During the stock out period or shortage time, there is complete backlogging.

\section{2. Notations}

The notations which are used to develop the inventory model are listed below. 
Tab. 1. Symbols and items which are used in the inventory model

\begin{tabular}{c|c||c|c}
\hline Symbols & Items & Symbols & Items \\
\hline (i) $I_{1}(t)$ & Positive inventory level at time $t$. & (ii) $I_{2}(t)$ & Negative inventory level at time $t$. \\
\hline (iii) $C_{1}$ & Holding cost per unit per unit time. & (iv) $C_{2}$ & Shortage cost per unit per unit time. \\
\hline (v) $T$ & Length of the ordering cycle. & (vi) $T C_{\alpha, \beta}^{a v}$ & $\begin{array}{c}\text { Total average cost per unit per unit time } \\
\text { with fractional effect. }\end{array}$ \\
\hline (vii) $T^{*}$ & Optimal ordering interval. & (viii) $T C^{*}$ & Minimized total average cost. \\
\hline (ix) $C_{3}$ & Ordering cost per unit per order. & (x) $(\Gamma,)$. & Gamma function \\
\hline (xi) $H C_{\alpha, \beta}$ & Inventory holding cost with memory effect. & (xii) $(B,)$. & Beta function \\
\hline (xiii) $P C_{\alpha, \beta}$ & Purchasing cost per unit time with memory & (xiv) $S C_{\alpha, \beta}$ & Shortage cost per unit with memory effect. \\
\hline (xv) $T_{\alpha, \beta}^{*}$ & $\begin{array}{c}\text { Optimal ordering interval with memory } \\
\text { effect. }\end{array}$ & (xvi) $T C_{\alpha, \beta}^{*}$ & $\begin{array}{c}\text { Minimized total average cost with memory } \\
\text { effect. }\end{array}$ \\
\hline
\end{tabular}

\section{3. Mathematical Formulation}

As the positive inventory level $I_{1}(t)$ reduces due to quadratic type demand during the time interval $\left[0, t_{1}\right]$ and negative inventory level $I_{2}(t)$ undergoes due to constant demand in the time interval $\left[t_{1}, T\right]$. During the positive inventory $\left[0, t_{1}\right]$ the demand rate is $\left(a+b t+c t^{2}\right)$ and during the shortage time interval $\left[t_{1}, T\right]$ demand rate is $D_{0}$. The classical inventory system is governed by the two ordinary differential equations as in the following form

$$
\begin{aligned}
& \frac{d\left(I_{1}(t)\right)}{d t}=-\left(a+b t+c t^{2}\right) \\
& \text { for } 0 \leq t \leq t_{1} \text { with } I_{1}\left(t_{1}\right)=0, \\
& \frac{d\left(I_{2}(t)\right)}{d t}=-\left(D_{0}\right) \\
& \text { for } t_{1} \leq t \leq T \text { with } I_{2}\left(t_{1}\right)=0 .
\end{aligned}
$$

We do not want to describe the derivation of the whole classical inventory model because our focus is to develop the memory dependent inventory model or fractional order inventory model.

\section{FRACTIONAL ORDER INVENTORY MODEL FORMULATION WITH MEMORY KERNEL}

To establish the influence of memory effects, first the differential equations $(10,11)$ can be written using the kernels function as follows [1]

$$
\begin{gathered}
\frac{d I_{1}(t)}{d t}=-\int_{0}^{t} k\left(t-t^{\prime}\right)\left(a+b t^{\prime}+c\left(t^{\prime}\right)^{2}\right) d t^{\prime} \\
\frac{d I_{2}(t)}{d t}=-\int_{0}^{t} k\left(t-t^{\prime}\right) D_{0} d t^{\prime}
\end{gathered}
$$

in which $k\left(t-t^{\prime}\right)$ is the kernel function. This type of kernel guarantees the existence of scaling features as it is often intrinsic in most natural phenomena. Thus, to generate the fractional order model we consider $k\left(t-t^{\prime}\right)=$ $\frac{1}{\Gamma(\alpha-1)}\left(t-t^{\prime}\right)^{\alpha-2}$ where $0<\alpha \leq 1$ and, $\Gamma(\alpha)$ denotes the gamma function.

Using the definition of fractional derivative $[9,10]$ we can re-write the Eq. $(12,13)$ to the form of fractional differential equations with the Caputo-type derivative in the following form

$$
\begin{gathered}
\frac{d I_{1}(t)}{d t}=-{ }_{0} D_{t}^{-(\alpha-1)}\left(a+b t+c t^{2}\right), \\
\frac{d I_{2}(t)}{d t}=-{ }_{0} D_{t}^{-(\alpha-1)}\left(D_{0}\right) .
\end{gathered}
$$

Now, applying fractional Caputo derivative of order $(\alpha-1)$ on both sides of the Eq. $(14,15)$ and using the fact the Caputo fractional order derivative and fractional order integral are inverse operators, the following fractional differential equations can be obtained for the model

$$
{ }_{0}^{\mathrm{C}} D_{t}^{\alpha}\left(I_{1}(t)\right)=-\left(a+b t+c t^{2}\right),
$$

$$
{ }_{0}^{\mathrm{C}} D_{t}^{\alpha}\left(I_{2}(t)\right)=-D_{0},
$$

along with boundary condition $I_{1}\left(t_{1}\right)=0, I_{2}\left(t_{1}\right)=0$.

\section{1. Analysis of the fractional order inventory model}

Thus the fractional order inventory model can be governed by the two fractional order differential equations in the following form as

$$
\begin{aligned}
& \frac{d^{\alpha}\left(I_{1}(t)\right)}{d t^{\alpha}}=-\left(a+b t+c t^{2}\right), \\
& \text { where } 0 \leq t \leq t_{1} \text { with } I_{1}\left(t_{1}\right)=0,
\end{aligned}
$$


$\frac{d^{\alpha}\left(I_{2}(t)\right)}{d t^{\alpha}}=-D_{0}$

where $t_{1} \leq t \leq T$ with $I_{2}\left(t_{1}\right)=0$,

( $\alpha$ is considered as differential memory index).

After solving the equation $(18,19)$, we get the inventory level in the following form:

$$
\begin{gathered}
I_{1}(t)=\frac{a}{\Gamma(1+\alpha)}\left(t_{1}^{\alpha}-t^{\alpha}\right)+ \\
+\frac{b}{\Gamma(2+\alpha)}\left(t_{1}^{\alpha+1}-t^{\alpha+1}\right)+\frac{2 c}{\Gamma(3+\alpha)}\left(t_{1}^{\alpha+2}-t^{\alpha+2}\right), \\
I_{2}(t)=\frac{D_{0}}{\Gamma(1+\alpha)}\left(t_{1}^{\alpha}-t^{\alpha}\right) .
\end{gathered}
$$

As the inventory level decreases with respect to time $t$, so the maximum positive inventory level is

$$
\begin{aligned}
M=I_{1}(0)= & \frac{a}{\Gamma(1+\alpha)}\left(t_{1}^{\alpha}\right)+\frac{b}{\Gamma(2+\alpha)}\left(t_{1}^{\alpha+1}\right)+ \\
& +\frac{2 c}{\Gamma(3+\alpha)}\left(t_{1}^{\alpha+2}\right) .
\end{aligned}
$$

The maximum backorder units during the shortage period is

$$
S=-I_{2}(T)=\left(\frac{D_{0}}{\Gamma(1+\alpha)}\left(T^{\alpha}-t_{1}^{\alpha}\right)\right) .
$$

Therefore, the order size during $[0, T]$, is denoted by $Q$ and defined as

$$
\begin{aligned}
Q= & (\text { maximum positive inventory level })+ \\
& +(\text { maximum backlogged demand } \\
& \text { during shortage period }))= \\
= & M+S=\left(\frac{a}{\Gamma(1+\alpha)}\left(t_{1}^{\alpha}\right)+\frac{b}{\Gamma(2+\alpha)}\left(t_{1}^{\alpha+1}\right)+\right. \\
& \left.+\frac{2 c}{\Gamma(3+\alpha)}\left(t_{1}^{\alpha+2}\right)\right)+\left(\frac{D_{0}}{\Gamma(1+\alpha)}\left(T^{\alpha}-t_{1}^{\alpha}\right)\right) .
\end{aligned}
$$

Inventory holding Cost with the memory effect is denoted by $H C_{\alpha, \beta}[6]$ and defined as

$$
\begin{aligned}
& H C_{\alpha, \beta}=C_{1} D^{-\beta}\left(I_{1}(t)\right)= \\
& =\frac{C_{1}}{\Gamma(\beta)} \int_{0}^{t_{1}}\left(t_{1}-t\right)^{(\beta-1)} I_{1}(t) d t= \\
& =\frac{C_{1}}{\Gamma(\beta)}\left(\frac{a t_{1}^{\alpha+\beta}}{\Gamma(1+\alpha)}\left(\left(\frac{1}{\beta}\right)-B(\alpha+1, \beta)\right)+\right. \\
& \quad+\frac{b t_{1}^{\alpha+\beta+1}}{\Gamma(2+\alpha)}\left(\left(\frac{1}{\beta}\right)-B(\alpha+2, \beta)\right)+ \\
& \left.\quad+\frac{2 c t_{1}^{\alpha+\beta+2}}{\Gamma(3+\alpha)}\left(\left(\frac{1}{\beta}\right)-B(\alpha+3, \beta)\right)\right),
\end{aligned}
$$

where $0 \leq t \leq t_{1}$ ( $\beta$ is considered as integral memory index ).
Shortage cost with the memory effect is denoted by $S C_{\alpha, \beta}$ and defined as follows

$$
\begin{aligned}
& S C_{\alpha, \beta}(T)=-C_{2 t_{1}} D_{T}^{-\beta}\left(I_{2}(t)\right)= \\
& =\frac{C_{2}}{\Gamma(\beta)} \int_{t_{1}}^{T}(T-t)^{(\beta-1)}\left(\frac{D_{0}}{\Gamma(1+\alpha)}\left(t^{\alpha}-t_{1}^{\alpha}\right)\right) d t= \\
& =-\frac{C_{2} D_{0} t_{1}^{\alpha}}{\Gamma(\beta+1) \Gamma(1+\alpha)} T^{\beta}+\frac{C_{2} D_{0} \alpha t_{1}^{\alpha+1}}{(1+\alpha) \Gamma(\beta) \Gamma(1+\alpha)} T^{\beta-1}+ \\
& \quad+\frac{C_{2} D_{0}}{\Gamma(\beta) \Gamma(1+\alpha)}\left(\frac{1}{(1+\alpha)}-\frac{(\beta-1)}{(2+\alpha)}\right) T^{\beta+\alpha}+ \\
& \quad+\frac{C_{2} D_{0}(\beta-1) t_{1}^{\alpha+2}}{(2+\alpha) \Gamma(\beta) \Gamma(1+\alpha)} T^{\beta-2},
\end{aligned}
$$

(expanding $(T-t)^{(\beta-1)} \cong T^{\beta-1}\left(1-(\beta-1) \frac{t}{T}\right)$, neglecting higher term as $\left.\left(\frac{t}{T}\right)\right)$.

Purchasing cost for the fractional order model is denoted by $P C_{\alpha, \beta}$ and defined

$$
\begin{aligned}
& P C_{\alpha, \beta}= P \\
& \times Q=P\left(\frac{a}{\Gamma(1+\alpha)}\left(t_{1}^{\alpha}\right)+\frac{b}{\Gamma(2+\alpha)}\left(t_{1}^{\alpha+1}\right)+\right. \\
&\left.+\frac{2 c}{\Gamma(3+\alpha)}\left(t_{1}^{\alpha+2}\right)+\frac{D_{0}}{\Gamma(1+\alpha)}\left(T^{\alpha}-t_{1}^{\alpha}\right)\right) .
\end{aligned}
$$

Therefore, total average cost for the fractional order inventory model is as

$$
\begin{aligned}
& T C_{\alpha, \beta}=\frac{\left(H C_{\alpha, \beta}+S C_{\alpha, \beta}+P C_{\alpha, \beta}+C_{3}\right)}{T}= \\
& =\left(H C_{\alpha, \beta}+C_{3}+P\left(\frac{a}{\Gamma(1+\alpha)}\left(t_{1}^{\alpha}\right)+\frac{b}{\Gamma(2+\alpha)}\left(t_{1}^{\alpha+1}\right)+\right.\right. \\
& \left.\left.+\frac{2 c}{\Gamma(3+\alpha)}\left(t_{1}^{\alpha+2}\right)\right)-\frac{P D_{0} t_{1}^{\alpha}}{\Gamma(1+\alpha)}\right) T^{-1}+\frac{P D_{0}}{\Gamma(1+\alpha)} T^{\alpha-1}+ \\
& -\frac{C_{2} D_{0} t_{1}^{\alpha}}{\beta \Gamma(\beta) \Gamma(\alpha+1)} T^{\beta-1}+\left(\frac{C_{2} D_{0} \alpha t_{1}^{\alpha+1}}{\Gamma(\beta) \Gamma(\alpha+1)(\alpha+1)}\right) T^{\beta-2}+ \\
& +\left(\frac{C_{2} D_{0}}{\Gamma(\beta) \Gamma(\alpha+1)(\alpha+1)}-\frac{C_{2} D_{0}(\beta-1)}{\Gamma(\beta) \Gamma(\alpha+1)(\alpha+2)}\right) T^{\alpha+\beta-1}+ \\
& +\frac{C_{2} D_{0}(\beta-1) t_{1}^{\alpha+2}}{(\alpha+2) \Gamma(\beta) \Gamma(\alpha+1)} T^{\beta-3} .
\end{aligned}
$$

We consider different cases of the total average cost depending on the different values of the memory indexes $\alpha, \beta$ : (i) $0<\alpha \leq 1.0,0<\beta \leq 1.0$. (ii) $\beta=1.0,0<\alpha \leq 1.0$. (iii) $\alpha=1.0,0<\beta \leq 1.0$.

(i) Case-1: $0<\alpha \leq 1.0,0<\beta \leq 1.0$.

In this case, the inventory model can be written as follows

$$
\left\{\begin{aligned}
\operatorname{Min} T C_{\alpha, \beta}(T)= & A T^{-1}+B_{1} T^{\alpha-1}+C T^{\beta-1}+ \\
& +D T^{\beta-2}+E T^{\alpha+\beta-1}+F T^{\beta-3} \\
\text { Subject to } T \geq 0 &
\end{aligned}\right.
$$


where, $A=P\left(\frac{a t_{1}^{\alpha}}{\Gamma(1+\alpha)}+\frac{b t_{1}^{\alpha+1}}{\Gamma(2+\alpha)}+\frac{2 c t_{1}^{\alpha+2}}{\Gamma(3+\alpha)}-\frac{D_{0} t_{1}^{\alpha}}{\Gamma(\alpha+1)}\right)+$ $+C_{3}+H C_{\alpha, \beta}, \quad C=-\frac{C_{2} D_{0} t_{1}^{\alpha}}{\beta \Gamma(\beta) \Gamma(\alpha+1)}, \quad B_{1}=\frac{P D_{0}}{\Gamma(\alpha+1)}$, $D=\frac{C_{2} D_{0} \alpha t_{1}^{\alpha+1}}{(\alpha+1) \Gamma(\beta) \Gamma(\alpha+1)}, E=\frac{C_{2} D_{0}}{\Gamma(\beta) \Gamma(\alpha+1)}\left(\frac{1}{(\alpha+1)}-\frac{(\beta-1)}{(\alpha+2)}\right)$, $F=\frac{C_{2} D_{0}(\beta-1) t_{1}^{\alpha+2}}{(\alpha+2) \Gamma(\beta) \Gamma(\alpha+1)}$.

\section{(a) Primal Geometric Programming Method}

To solve (28) analytically, the primal geometric programming method has been applied. The dual form of (28) has been introduced by the dual variable $(w)$. The corresponding primal geometric programming problem has been constructed in the following form as

$\operatorname{Max} d(w)=\left(\frac{A}{w_{1}}\right)^{w_{1}}\left(\frac{B_{1}}{w_{2}}\right)^{w_{2}}\left(\frac{C}{w_{3}}\right)^{w_{3}}\left(\frac{D}{w_{4}}\right)^{w_{4}}\left(\frac{E}{w_{5}}\right)^{w_{5}}\left(\frac{F}{w_{6}}\right)^{w_{6}}$.

Normalized condition is as

$$
w_{1}+w_{2}+w_{3}+w_{4}+w_{5}+w_{6}=1
$$

Orthogonal condition is as

$$
\begin{aligned}
& -w_{1}+(\alpha-1) w_{2}+(\beta-1) w_{3}+(\beta-2) w_{4}+ \\
& +(\alpha+\beta-1) w_{5}+(\beta-3) w_{6}=0
\end{aligned}
$$

and the primal-dual relations are as follows

$$
\left.\begin{array}{l}
A T^{-1}=w_{1} d(w), \quad B_{1} T^{\alpha-1}=w_{2} d(w) \\
C T^{\beta-1}=w_{3} d(w), \quad D T^{\beta-2}=w_{4} d(w) \\
E T^{\alpha+\beta-1}=w_{5} d(w), \quad F T^{\beta-3}=w_{6} d(w)
\end{array}\right\} .
$$

Using the above primal-dual relations the followings are evaluated as

$$
\begin{aligned}
& \frac{A w_{2}}{B_{1} w_{1}}=\left(\frac{D w_{3}}{C w_{4}}\right)^{\alpha}, \frac{C w_{2}}{B_{1} w_{3}}=\left(\frac{D w_{3}}{C w_{4}}\right)^{\alpha-\beta}, \\
& \frac{D w_{5}}{E w_{4}}=\left(\frac{D w_{3}}{C w_{4}}\right)^{\alpha+1}, \frac{F w_{5}}{E w_{6}}=\left(\frac{D w_{3}}{C w_{4}}\right)^{\alpha+2},
\end{aligned}
$$

along with

$$
T=\frac{D w_{3}}{C w_{4}}
$$

Solving (30), (31) and (33) the critical value $w_{1}^{*}, w_{2}^{*}, w_{3}^{*}$, $w_{4}^{*}, w_{5}^{*}, w_{6}^{*}$ of the dual variables $w_{1}, w_{2}, w_{3}, w_{4}, w_{5}, w_{6}$ can be obtained and finally the optimum value $T^{*}$ of $T$ has been calculated from the equation of (34) substituting the critical values. Now the minimized total average cost $T C_{\alpha, \beta}^{*}$ has been calculated by substituting $T^{*}$ in (28) analytically. The minimized total average cost and the optimal ordering interval is evaluated from (28) numerically. (ii) Case-2: $\beta=1.0,0<\alpha \leq 1.0$.

In this case, the inventory model is

$$
\left\{\begin{array}{rl}
\operatorname{Min} T C_{\alpha, 1}(T)= & A T^{-1}+B_{1} T^{\alpha-1}+C T^{0}+ \\
& +D T^{-1}+E T^{\alpha}+F T^{-2} \\
\text { Subject to } T \geq 0 & 0
\end{array}\right.
$$

where, $A=P\left(\frac{a t_{1}^{\alpha}}{\Gamma(1+\alpha)}+\frac{b t_{1}^{\alpha+1}}{\Gamma(2+\alpha)}+\frac{2 c t_{1}^{\alpha+2}}{\Gamma(3+\alpha)}-\frac{D_{0} t_{1}^{\alpha}}{\Gamma(\alpha+1)}\right)+C_{3}+$ $+H C_{\alpha, 1}, B_{1}=\frac{P D_{0}}{\Gamma(\alpha+1)}, C=-\frac{C_{2} D_{0} t_{1}^{\alpha}}{\Gamma(\alpha+1)}, D=\frac{C_{2} D_{0} \alpha t_{1}^{\alpha+1}}{(\alpha+1) \Gamma(\alpha+1)}$, $E=\frac{C_{2} D_{0}}{(\alpha+1) \Gamma(\beta) \Gamma(\alpha+1)}, F=0$.

Using the similar analogy as previously, the minimized total average cost and optimal ordering interval has been solved from (35).

(iii) Case-3: $\alpha=1.0, \quad 0<\beta \leq 1.0$.

In this case, the inventory model becomes,

$$
\left\{\begin{array}{l}
\operatorname{Min} T C_{1, \beta}(T)=A T^{-1}+B_{1} T^{0}+C T^{\beta-1}+ \\
\quad+D T^{\beta-2}+E T^{\beta}+F T^{\beta-3} \\
\text { Subject to } T \geq 0
\end{array}\right.
$$

where, $A=P\left(\frac{a t_{1}}{\Gamma(2)}+\frac{b t_{1}^{2}}{\Gamma(3)}+\frac{2 c t_{1}^{3}}{\Gamma(4)}-\frac{D_{0} t_{1}}{\Gamma(2)}\right)+C_{3}+H C_{1, \beta}$, $B_{1}=\frac{P D_{0}}{\Gamma(2)}, C=-\frac{C_{2} D_{0} t_{1}}{\beta \Gamma(\beta) \Gamma(2)}, D=\frac{C_{2} D_{0} t_{1}^{2}}{2 \Gamma(\beta) \Gamma(2)}, E=\frac{C_{2} D_{0}}{\Gamma(\beta) \Gamma(2)} \times$ $\times\left(\frac{1}{2}-\frac{(\beta-1)}{3}\right), F=\frac{C_{2} D_{0}(\beta-1) t_{1}^{3}}{3 \Gamma(\beta) \Gamma(2)}$.

Using the similar way of Case-1, the minimized total average cost and the optimal ordering interval has been solved from (36).

\section{NUMERICAL EXAMPLES}

(i) To establish the memory effect in the inventory model, we have found the optimal ordering interval $\left(T_{\alpha, \beta}^{*}\right)$, minimized total average cost $\left(T C_{\alpha, \beta}^{*}\right)$ for different values of the memory index $\alpha, \beta$ considering the model parameters as given in Tab. 2.

Tab. 2. Fixed values of the parameter in the numerical example

\begin{tabular}{l|c|c|c|c|c|c|c|c|c}
\hline Parameter & $P$ & $C_{3}$ & $C_{1}$ & $C_{2}$ & $D_{0}$ & $a$ & $b$ & $c$ & $t_{1}$ \\
\hline Value & 8 & 250 & 0.5 & 1.0 & 15 & 2 & 6 & 13 & 0.3456 \\
\hline
\end{tabular}

It is observed from Tab. 3 that when memory of the system is gradually increasing, minimized total average cost is gradually decreasing, i.e. profit is high in long memory effect compared to short memory effect. In long memory effect, minimized total average cost is very low compared 
Tab. 3. Optimal ordering interval and minimized total average cost for $\beta=1.0$, and $\alpha$ varies from 0.1 to 1.0 as defined in Sec. IV.1

\begin{tabular}{c|c|c|c}
\hline$\alpha$ & $\beta$ & $T_{\alpha, \beta}^{*}$ & $T C_{\alpha, \beta}^{*}$ \\
\hline $\mathbf{0 . 1}$ & $\mathbf{1 . 0}$ & $\begin{array}{c}\mathbf{6 7 9 . 3 7 9 8} \\
\text { (maximum value) }\end{array}$ & $\mathbf{1 6 . 1 2 4 3}$ \\
\hline $\begin{array}{c}0.2 \uparrow \text { (growing } \\
\text { memory effect) }\end{array}$ & 1.0 & 230.6127 & 35.6275 \\
\hline $0.3 \uparrow$ & 1.0 & 114.7478 & 58.6401 \\
\hline $0.4 \uparrow$ & 1.0 & 68.0305 & 84.6849 \\
\hline $0.5 \uparrow$ & 1.0 & 44.6124 & 113.2369 \\
\hline $0.6 \uparrow$ & 1.0 & 31.1730 & 143.6906 \\
\hline $0.7 \uparrow$ & 1.0 & 22.7070 & 175.2772 \\
\hline $0.8 \uparrow$ & 1.0 & 17.0060 & 206.9958 \\
\hline $0.9 \uparrow$ & 1.0 & 12.9827 & $\begin{array}{c}237.5986 \downarrow \\
(\text { decreasing) }\end{array}$ \\
\hline $\mathbf{1 . 0}$ & $\mathbf{1 . 0}$ & $\begin{array}{c}\mathbf{1 0 . 0 5 6 6} \uparrow \\
\text { (increasing) }\end{array}$ & $\mathbf{2 6 5 . 6 6 4 7}$ \\
\hline
\end{tabular}

to short memory effect or memory less system. In long memory effect, business takes a long time to reach the minimum value of the total average cost compared to low memory effect or memory less system.

(ii) Consider $c=0$ keeping the other parameters fixed as in the Tab. 2, i.e. demand rate is linear type with initial demand.

Tab. 4. Optimal ordering interval and minimized total average cost for $\beta=1.0$, and $\alpha$ varies from 0.1 to 1.0 as defined in Sec. IV.1

\begin{tabular}{c|c|c|c}
\hline$\alpha$ & $\beta$ & $T_{\alpha, \beta}^{*}$ & $T C_{\alpha, \beta}^{*}$ \\
\hline $\mathbf{0 . 1}$ & $\mathbf{1 . 0}$ & $\begin{array}{c}\mathbf{1 4 9 . 6 3 5 4} \\
\text { (maximum value) }\end{array}$ & $\mathbf{1 1 . 9 7 8 1}$ \\
\hline $\begin{array}{c}0.2 \uparrow \text { (growing } \\
\text { memory effect) }\end{array}$ & 1.0 & 65.6815 & 25.4371 \\
\hline $0.3 \uparrow$ & 1.0 & 39.5173 & 41.2693 \\
\hline $0.4 \uparrow$ & 1.0 & 26.9637 & 59.5881 \\
\hline $0.5 \uparrow$ & 1.0 & 19.5944 & 80.2670 \\
\hline $0.6 \uparrow$ & 1.0 & 14.7296 & 102.9165 \\
\hline $0.7 \uparrow$ & 1.0 & 11.2780 & 126.8481 \\
\hline $0.8 \uparrow$ & 1.0 & 8.7276 & 151.0789 \\
\hline $0.9 \uparrow$ & 1.0 & 6.8151 & 174.1198 \\
\hline $\mathbf{1 . 0}$ & $\mathbf{1 . 0}$ & $\begin{array}{c}\mathbf{5 . 3 9 0 4} \uparrow \\
\text { (increasing) }\end{array}$ & $\begin{array}{c}\mathbf{1 9 5 . 6 7 1 6} \downarrow \\
\text { (decreasing above) }\end{array}$ \\
\hline
\end{tabular}

It is found from Tab. 4 that the same nature of changes of minimized total average cost and optimal ordering interval has been found like Tab. 3 but the numerical value is low compared to Tab. 3 . (iii) Consider $a=0$ keeping other parameters fixed as in the Tab. 2 , i.e. quadratic type demand rate with no initial demand.

Tab. 5. Optimal ordering interval and minimized total average cost for $\beta=1.0$, and $\alpha$ varies from 0.1 to 1.0 as defined in Sec. IV.1

\begin{tabular}{c|c|c|c}
\hline$\alpha$ & $\beta$ & $T_{\alpha, \beta}^{*}$ & $T C_{\alpha, \beta}^{*}$ \\
\hline $\mathbf{0 . 1}$ & $\mathbf{1 . 0}$ & $\begin{array}{c}\mathbf{6 7 4 . 3 1 7 7} \\
\text { (maximum value) }\end{array}$ & $\mathbf{1 6 . 1 0 2 0}$ \\
\hline $\begin{array}{c}0.2 \uparrow \text { (growing } \\
\text { memory effect) }\end{array}$ & 1.0 & 229.1122 & 35.5660 \\
\hline $0.3 \uparrow$ & 1.0 & 114.0888 & 58.5263 \\
\hline $0.4 \uparrow$ & 1.0 & 67.6811 & 84.5101 \\
\hline $0.5 \uparrow$ & 1.0 & 44.4043 & 112.9967 \\
\hline $0.6 \uparrow$ & 1.0 & 31.0388 & 143.3838 \\
\hline $0.7 \uparrow$ & 1.0 & 22.6150 & 174.9045 \\
\hline $0.8 \uparrow$ & 1.0 & 16.9400 & 206.5590 \\
\hline $0.9 \uparrow$ & 1.0 & 12.9338 & 237.1002 \\
\hline $\mathbf{1 . 0}$ & $\mathbf{1 . 0}$ & $\begin{array}{c}\mathbf{1 0 . 0 1 9 5} \uparrow \\
\text { (increasing) }\end{array}$ & $\begin{array}{c}\mathbf{2 6 5 . 1 0 7 9} \downarrow \\
\text { (decreasing above) }\end{array}$ \\
\hline
\end{tabular}

It is observed from Tab. 5 that minimized total average cost is gradually decreasing with gradually increasing memory effect but the numerical value of the optimal ordering interval is gradually increasing with gradually increasing memory effect. The numerical values of the minimized total average cost and optimal ordering interval are low compared to Tab. 3 .

(iv) Consider $b=0$ keeping other parameters fixed as in the Tab. 2, i.e. demand rate is square of the time with initial demand.

Tab. 6. Optimal ordering interval and minimized total average cost for $\beta=1.0$, and $\alpha$ varies from 0.1 to 1.0 as defined in Sec. IV.1

\begin{tabular}{c|c|c|c}
\hline$\alpha$ & $\beta$ & $T_{\alpha, \beta}^{*}$ & $T C_{\alpha, \beta}^{*}$ \\
\hline $\mathbf{0 . 1}$ & $\mathbf{1 . 0}$ & $\begin{array}{c}\mathbf{6 7 4 . 5 6 4 1} \\
\text { (maximum value) }\end{array}$ & $\begin{array}{c}\mathbf{1 6 . 1 0 3 1} \\
\text { (minimum value) }\end{array}$ \\
\hline $\begin{array}{c}0.2 \uparrow \text { (growing } \\
\text { memory effect) }\end{array}$ & 1.0 & 229.3058 & 35.5739 \\
\hline $0.3 \uparrow$ & 1.0 & 114.2185 & 58.5487 \\
\hline $0.4 \uparrow$ & 1.0 & 67.7702 & 84.5547 \\
\hline $0.5 \uparrow$ & 1.0 & 44.4678 & 113.0701 \\
\hline $0.6 \uparrow$ & 1.0 & 31.0856 & 143.4910 \\
\hline $0.7 \uparrow$ & 1.0 & 22.6507 & 175.0490 \\
\hline $0.8 \uparrow$ & 1.0 & 16.9679 & 206.7433 \\
\hline $0.9 \uparrow$ & 1.0 & 12.9559 & 237.3258 \\
\hline $\mathbf{1 . 0}$ & $\mathbf{1 . 0}$ & $\begin{array}{c}\mathbf{1 0 . 0 3 7 3} \uparrow \\
\text { (increasing) }\end{array}$ & $\begin{array}{c}\mathbf{2 6 5 . 3 7 5 3} \downarrow \\
\text { (decreasing above) }\end{array}$ \\
\hline
\end{tabular}


It is observed from the Tab. 6, that in long memory effect (here $\alpha=0.1$ ), minimized total average cost is very low compared to the short memory effect or memory less system. In long memory effect, business stay long time compared to the short memory effect. The numerical value of the minimized total average cost and optimal ordering interval are low compared to the Tab. 3 but more compared to the Tab. $4,5$.

(v) Consider $a=0, b=0$ keeping other parameters fixed as in Tab. 2, i.e. demand rate is square of the time proportional.

Tab. 7. Optimal ordering interval and minimized total average cost for $\beta=1.0$, and $\alpha$ varies from 0.1 to 1.0 as defined in Sec. IV.1

\begin{tabular}{c|c|c|c}
\hline$\alpha$ & $\beta$ & $T_{\alpha, \beta}^{*}$ & $T C_{\alpha, \beta}^{*}$ \\
\hline $\mathbf{0 . 1}$ & $\mathbf{1 . 0}$ & $\begin{array}{c}\mathbf{6 6 9 . 4 9 7 9} \\
\text { (maximum value) }\end{array}$ & $\begin{array}{c}\mathbf{1 6 . 0 8 0 5} \\
\text { (minimum value) }\end{array}$ \\
\hline $\begin{array}{c}0.2 \uparrow \text { (growing } \\
\text { memory effect) }\end{array}$ & 1.0 & 227.8003 & 35.5121 \\
\hline $0.3 \uparrow$ & 1.0 & 113.5585 & 58.4343 \\
\hline $0.4 \uparrow$ & 1.0 & 67.4202 & 84.3792 \\
\hline $0.5 \uparrow$ & 1.0 & 44.2594 & 112.8291 \\
\hline $0.6 \uparrow$ & 1.0 & 30.9511 & 143.1833 \\
\hline $0.7 \uparrow$ & 1.0 & 22.5585 & 174.6755 \\
\hline $0.8 \uparrow$ & 1.0 & 16.9018 & 206.3056 \\
\hline $0.9 \uparrow$ & 1.0 & 12.9069 & 236.8263 \\
\hline $\mathbf{1 . 0}$ & $\mathbf{1 . 0}$ & $\begin{array}{c}\mathbf{1 0 . 0 0 0 1} \uparrow \\
\text { (increasing) }\end{array}$ & $\begin{array}{c}\mathbf{2 6 4 . 8 1 7 4} \downarrow \\
\text { (decreasing above) }\end{array}$ \\
\hline
\end{tabular}

It is found from Tab. 7, in long memory effect or short memory effect, the nature of the changing of the minimized total average cost is same like Tab. 3, 5, 6 but the numerical value is low compared to Tab. 3, 5, 6 .

(vi) Consider $a=0, c=0$ keeping other parameters fixed as in Tab. 2, i.e. demand rate is time proportional with no initial demand.

Tab. 8 shows that the nature of changing of the minimized total average cost and the optimal ordering interval are the same as Tab. 3-7 but the numerical values are low compared to Tab. 3-7, 9 .

(vii) Consider $b=0, c=0$ keeping other parameters fixed as in Tab. 2, i.e demand rate is constant which was initially established.

Tab. 9 clears that the nature of changing of the minimized total average cost and optimal ordering interval are the same, i.e. they are sequentially decreasing with respect to memory index as Tab. 3-8 but the numerical values are low compared to Tab. 3-7 except Tab. 8 .

Hence, it is observed from Tab. 3-9 that when the demand rate is the proportional time the numerical value of the minimized total average cost is low compared to the other
Tab. 8. Optimal ordering interval and minimized total average cost for $\beta=1.0$, and $\alpha$ varies from 0.1 to 1.0 as defined in Sec. IV.1

\begin{tabular}{c|c|c|c}
\hline$\alpha$ & $\beta$ & $T_{\alpha, \beta}^{*}$ & $T C_{\alpha, \beta}^{*}$ \\
\hline $\mathbf{0 . 1}$ & $\mathbf{1 . 0}$ & $\begin{array}{c}\mathbf{1 4 3 . 4 9 7 5} \\
\text { (maximum value) }\end{array}$ & $\begin{array}{c}\mathbf{1 1 . 8 7 4 7} \\
\text { (minimum value) }\end{array}$ \\
\hline $\begin{array}{c}0.2 \uparrow \text { (growing } \\
\text { memory effect) }\end{array}$ & 1.0 & 63.5948 & 25.2183 \\
\hline $0.3 \uparrow$ & 1.0 & 38.5067 & 40.9354 \\
\hline $0.4 \uparrow$ & 1.0 & 26.3886 & 59.1434 \\
\hline $0.5 \uparrow$ & 1.0 & 19.2334 & 79.7164 \\
\hline $0.6 \uparrow$ & 1.0 & 14.4869 & 102.2633 \\
\hline $0.7 \uparrow$ & 1.0 & 11.1065 & 126.0936 \\
\hline $0.8 \uparrow$ & 1.0 & 8.6022 & 150.2233 \\
\hline $0.9 \uparrow$ & 1.0 & 6.7217 & 173.4655 \\
\hline $\mathbf{1 . 0}$ & $\mathbf{1 . 0}$ & $\begin{array}{c}\mathbf{5 . 2 0 8} \uparrow \\
\text { (increasing) }\end{array}$ & $\begin{array}{c}\mathbf{1 9 4 . 6 2 8 0} \downarrow \\
\text { (decreasing above) }\end{array}$ \\
\hline
\end{tabular}

Tab. 9. Optimal ordering interval and minimized total average cost for $\beta=1.0$, and $\alpha$ varies from 0.1 to 1.0 as defined in Sec. IV.1

\begin{tabular}{c|c|c|c}
\hline$\alpha$ & $\beta$ & $T_{\alpha, \beta}^{*}$ & $T C_{\alpha, \beta}^{*}$ \\
\hline $\mathbf{0 . 1}$ & $\mathbf{1 . 0}$ & $\begin{array}{c}\mathbf{1 4 3 . 7 9 7 1} \\
\text { (maximum value) }\end{array}$ & $\begin{array}{c}\mathbf{1 1 . 8 7 9 9} \\
\text { (minimum value) }\end{array}$ \\
\hline $\begin{array}{c}0.2 \uparrow \text { (growing } \\
\text { memory effect) }\end{array}$ & 1.0 & 63.8650 & 25.2469 \\
\hline $0.3 \uparrow$ & 1.0 & 38.7065 & 41.0017 \\
\hline $0.4 \uparrow$ & 1.0 & 26.5358 & 59.2576 \\
\hline $0.5 \uparrow$ & 1.0 & 19.3440 & 79.8854 \\
\hline $0.6 \uparrow$ & 1.0 & 14.5719 & 102.4923 \\
\hline $0.7 \uparrow$ & 1.0 & 11.1732 & 126.3872 \\
\hline $0.8 \uparrow$ & 1.0 & 8.6553 & 150.5855 \\
\hline $0.9 \uparrow$ & 1.0 & 6.7641 & 173.8986 \\
\hline $\mathbf{1 . 0}$ & $\mathbf{1 . 0}$ & $\begin{array}{c}\mathbf{5 . 3 5 4 3} \uparrow \\
\text { (increasing) }\end{array}$ & $\begin{array}{c}\mathbf{1 9 5 . 1 3 0 3} \downarrow \\
\text { (decreasing) }\end{array}$ \\
\hline
\end{tabular}

assumed demand rate like quadratic type demand rate, constant demand rate, square of the time proportional etc. Hence for time proportional demand rate, business gains more profit without risk because business does not take too long to reach the minimum value of the total average cost as well as minimized total average cost is low.

\section{GRAPHICAL PRESENTATION}

(a) Graphical comparison between positive inventory level for long memory affected system as well as low 


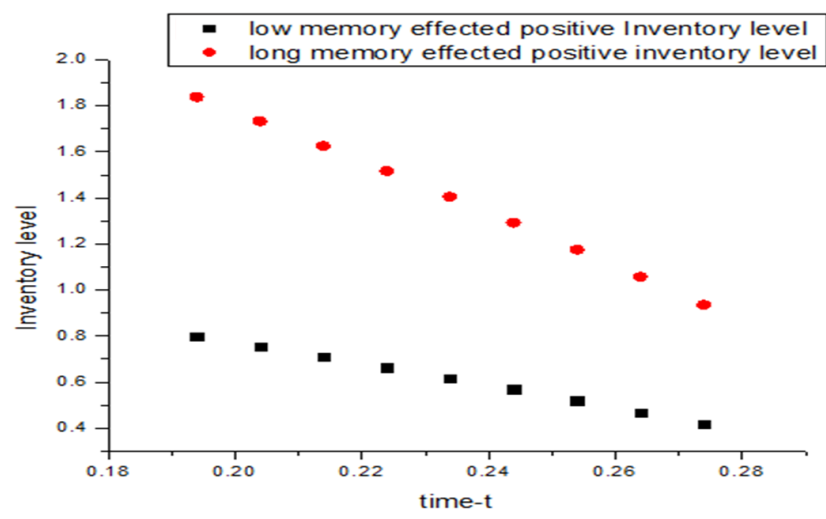

Fig. 2. Scatter diagram inventory level versus time corresponding low memory effect $(\alpha=0.9)$ and long memory effect $(\alpha=0.1)$ of the system

memory affected system with respect to time is presented in Fig. 2.

(b) Graphical comparison of the minimized total average cost for different cases as found numerically in the above tables are presented in Fig. 3.

From Fig. 2, it is observed that in long memory effect $(\alpha=0.1)$ inventory level is high but in low memory effect $(\alpha=0.9)$ inventory level is low comparatively long memory effect $(\alpha=0.1)$. It is also noted that Inventory level with respect to time is decreasing gradually for both cases for long memory effect as well as low memory effect.

From the above Fig. 3, it is observed that for some cases, minimized total average cost, which is very close, goes through one line, and for other cases minimized total average cost, which is very close, goes through another line.

\section{CONCLUSIONS}

In this paper, we have established the memory effect in an inventory system via fractional calculus. Here, we have considered a memory dependent inventory model with quadratic type demand rate and shortage. The demand rate of the cosmetic product increases with time during the seasonal period like Vaseline, ponds, Olive-oil during winter season and Body spray, perfume, etc., during the summer season. The demand rate most of the time practically increases with time, which may be proportional to time or square of the time, etc. Tab. 3-9 reveal when the system has no initial demand and the demand rate is proportional to the time, profit is maximum. One important observation is found that in long memory effect, business takes more time to reach the minimum value of the total average cost compared to the short memory effect. The inventory level with respect to time is plotted corresponding long memory affected system and short memory affected system. This plot (Fig. 2) presents that the inventory level for high memory affected system as well as low memory affected system is totally different as inventory level for low memory affected system is low but for

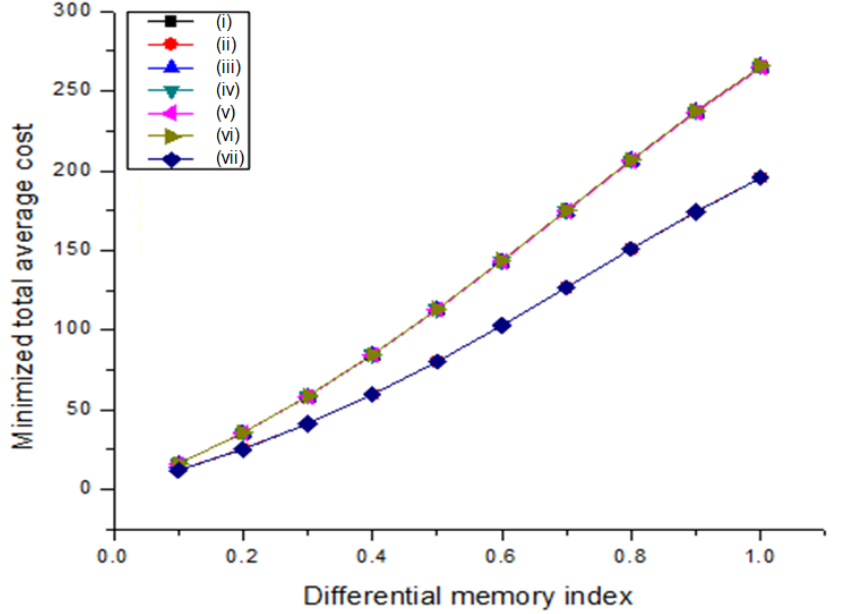

Fig. 3. Scatter diagram for memory index $\alpha$ versus minimized total average cost as numerically described in example (i)-(vii)

long memory affected system, inventory level is high. This paper may be extended for time delay in payment with memory effect. More new thoughts towards the memory dependent inventory model may be improved considering practical data from industries.

\section{Acknowledgment}

The authors would like to thank the reviewers and the editor for the valuable comments and suggestions. The first author would also like to thank the Department of Science and Technology, Government of India, New Delhi, for the financial assistance under AORC, Inspire fellowship Scheme towards this research work.

\section{References}

[1] M. Saeedian, M. Khalighi, N. Azimi-Tafreshi, G.R. Jafari, M. Ausloos, Memory effects on epidemic evolution: The susceptible-infected-recovered epidemic model, Physical Review E 95, 022409 (2017).

[2] V.V. Tarasova, V.E. Tarasov, Memory effects in hereditary Keynesian model, Problems of Modern Science and Education 38(80), 38-44 (2016). DOI: 10.20861/2304-2338-201680-001 [in Russian].

[3] V.E. Tarasov, V.V. Tarasova, Long and short memory in economics: fractional-order difference and differentiation, IRAInternational Journal of Management and Social Sciences 5(2), 327-334 (2016). DOI: 10.21013/jmss.v5.n2.p10.

[4] V.E. Tarasov, V.V. Tarasova, Economic interpretation of fractional derivatives, Progress in Fractional Differential and Applications 3(1), 1-6 (2017).

[5] V.E. Tarasov, V.V. Tarasova, Elasticity for economic processes with memory: fractional differential calculus approach, Fractional Differential Calculus 6(2), 219-232 (2016).

[6] R. Pakhira, U. Ghosh, S. Sarkar, Study of Memory Effects in an Inventory Model Using Fractional Calculus, Applied Mathematical Sciences 12(17), 797-824 (2018).

[7] R. Pakhira, U. Ghosh, S. Sarkar, Application of Memory effects In an Inventory Model with Linear Demand and No shortage, International Journal of Research in Advent Technology 6(8) (2018). 
[8] T. Das, U. Ghosh, S. Sarkar, S. Das, Time independent fractional Schrodinger equation for generalized Mie-type potential in higher dimension framed with Jumarie type fractional derivative, Journal of Mathematical Physics 59, 022111 (2018). DOI: 10.1063/1.4999262.

[9] K.S. Miller, B. Ross, An Introduction to the Fractional Calculus and Fractional Differential Equations, John Wiley \& Sons, New York, USA (1993).

[10] I. Podubly, Fractional Differential Equations, Mathematics in Science and Engineering, Academic Press, San Diego, California, USA (1999).

[11] F.W. Harris, Operations and Cost, 48-54, A. W. Shaw Company, Chicago (1915).

[12] R.H. Wilson, A Scientific Routine for Stock Control, Harvard Business Review 13(1), 116-128 (1934).

[13] N. Rajeswari, T. Vanjikkodi, An Inventory model for Items with Two Parameter Weibull Distribution Deterioration and Backlogging, American Journal of Operations Research 2, 247-252 (2012).

[14] E.A. Silver, A Simple inventory replenishment decision rule for a linear trend in demand, Journal of the Operational Research Society 30, 71-75 (1979).

[15] M. Deb, K. Chaudhuri, A note on the heuristic for replenishment of trended inventories considering shortages, J. Oper. Research Soc. 38, 459-463 (1987).

[16] E. Ritchie, The optimal EOQ for linear increasing demand: a simple optimal solution, Journal of the Operational Research Society 35, 949-952 (1984).

[17] U. Ghosh, S. Sengupta, S. Sarkar, S. Das, Characterization of non-differentiable points of a function by Fractional deriva- tive of Jumarie type, European Journal of Academic Essays 2(2), 70-86 (2015).

[18] U. Ghosh, S. Sengupta, S. Sarka, S. Das, Analytic Solution of linear fractional differential equation with Jumarie derivative in term of Mittag-Leffler function, American Journal of Mathematical Analysis 3(2), 32-38 (2015).

[19] S. Das, Functional Fractional Calculus for system Identification and Controls, Springer, Berlin, Heidelberg, New York (2008).

[20] A.K. Ojha, K.K. Biswal, Posynomial Geometric Programming Problems with Multiple parameters, Journal of Computing 2(1) (2010).

[21] R. Pakhira, U. Ghosh, S. Sarkar, Study of Memory Effect in an Inventory Model with Linear Demand and Salvage Value, International Journal of Applied Engineering Research 13(20), 14741-14751 (2018).

[22] R. Pakhira, U. Ghosh, S. Sarkar, Study of Memory Effect in Inventory Model with Quadratic Type Demand Rate and Salvage Value, Applied Mathematical Sciences 13(5), 209-223 (2019). DOI: 10.12988/ams.2019.9111.

[23] R. Pakhira, U. Ghosh, S. Sarkar, Study of Memory Effect In an Inventory Model with Linear Demand and Shortage, International Journal of Mathematical Sciences and Computing (IJMSC) (2019).

[24] R. Pakhira, U. Ghosh, S. Sarkar, Application of memory effect in an inventory model with price dependent demand rate during shortage, I.J. Education and Management Engineering (2019).

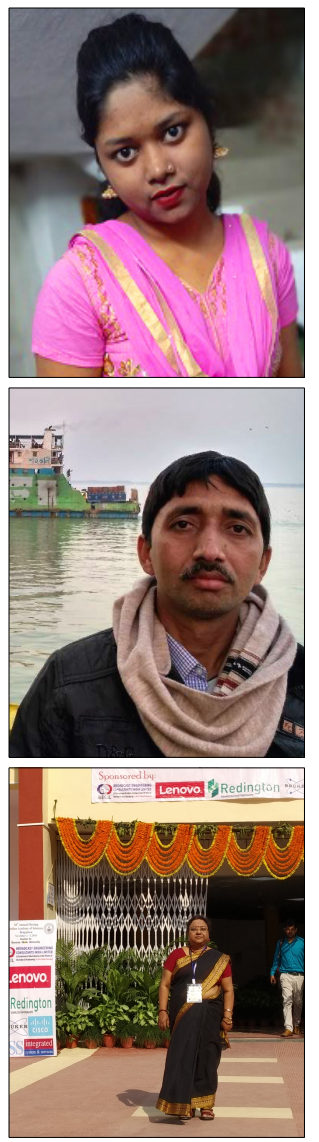

Rituparna Pakhira is currently P.HD DST-INSPIRE Research fellow Department of Applied Mathematics, University of Calcutta, West Bengal India. Kolkata, India. She obtained her MSc in Mathematics from Jadavpur University, West Bengal India in 2015. She is mainly interested in application of fractional calculus different topic of operation research. She has 6 publications in different international journals.

Uttam Ghosh, Assistant Professor of Applied Mathematics in University of Calcutta. His research field includes fractal geometry, information theory, percolation theory, biomathematics and fractional calculus. He has 43 publications in reputed national and international journals.

Susmita Sarkar is Professor of Applied Mathematics, University of Calcutta. Her field of research includes plasma dynamics, biomathematics and fractional calculus. She has more than 79 research publications in reputed international journals. Dr. Susmita Sarkar was TWAS Associate and ICTP regular Associate. 